\title{
MAC-Centric Cross-Layer Collaboration: A Case Study on Physical-Layer Network Coding
}

\author{
Qingyang Song ${ }^{1}$, Lei Guo ${ }^{1}$, Fanzhao Wang ${ }^{1}$, Shiqiang Wang ${ }^{2}$, and Abbas Jamalipour ${ }^{3}$ \\ ${ }^{1}$ School of Information Science and Engineering, Northeastern University, China \\ ${ }^{2}$ Department of Electrical and Electronic Engineering, Imperial College London, United Kingdom \\ ${ }^{3}$ School of Electrical and Information Engineering, University of Sydney, Australia
}

\begin{abstract}
Some newly emerging physical-layer cooperative communication techniques (e.g. physical-layer network coding (PNC), interference alignment, and virtual multiple-input multiple-output (VMIMO) scheme) provide high spectral efficiency and have attracted much attention. To implement these physical-layer techniques, the support from appropriate upper-layer mechanisms is required. This article takes PNC as an example to show how an upper layer mechanism can work appropriately to support PNC in a multi-hop network. We present a media access control (MAC) centric cross-layer collaboration mechanism for PNC, aiming at scheduling different relaying methods adaptively and allocating the correspondingly needed resources (e.g. transmission powers and channels) efficiently. Our numerical results affirm the efficiency of this mechanism, highlighting the importance of cross-layer collaboration for the implementation of physical-layer cooperative communication techniques.
\end{abstract}

\section{INTRODUCTION}

In wireless communications, unreliable radio channel conditions and limited wireless spectrum resources have become the bottleneck for high-speed data transmission. Cooperative communication has been considered as one of the most effective solutions to this growing and serious problem, where each node shares its hardware, processing, and energy resources to help each other in information delivery. The cooperative relaying technique extends the network coverage and increases its capacity, and has been standardized in the Third Generation Partnership Project (3GPP) Long Term Evolution (LTE)-Advanced and IEEE $802.16 \mathrm{~m}$.

The cooperation among nodes has been applied in both physical and higher layers. Particularly, some new physicallayer techniques, such as physical-layer network coding (PNC), interference alignment, and virtual multiple-input multiple-output (VMIMO) scheme, have attracted great interest and are considered as promising techniques for cooperative communications. However, the cooperation gain from any physical layer technique is closely related to the application scenario, network topology, traffic patterns, scheduling strategies, etc. The system performance cannot be improved if resources (e.g. channels and transmission powers) are assigned improperly by the upper layers. Therefore, multiple layers (including physical, media access control (MAC), and network layers) should be coupled in an optimization framework to support these physical-layer techniques. For a simple form of cooperative communications, there exist at least two senders and each of them is equipped with at least one antenna. PNC with two data flows just satisfies the minimum requirements. Therefore, we take it as an example to present how different layers collaborate to adaptively utilize cooperative communications in wireless multi-hop networks.

As a popular cooperative communication technique, network coding allows relay nodes to combine their received packets from independent traffic flows and then forward the combinations to neighbors, instead of forwarding each packet individually. This leads to fewer transmissions, and thus potentially improves spectrum utilization and network throughput. PNC further boosts channel capacity by utilizing the superposition of electromagnetic waves in the physical layer [1].

To make PNC applicable in practical systems, efforts on developing upper-layer mechanisms for PNC have been made. A PNC-based energy efficient routing scheme was recently proposed in [2], where each node is able to intelligently decide whether it should use PNC or point-topoint transmission to the next hop in order to minimize power consumption. A basic MAC protocol for simple topologies using PNC was proposed in [3], which was implemented on a software-defined radio prototype. In that protocol, a tail, which contains the same information as the header, is added to the packet, so that the header information can be successfully decoded from the nonsuperposed part of the superposed packet. A theoretical MAC protocol for PNC using the abstract MAC layer specification was proposed in [4]. In [5], a distributed MAC protocol for PNC (named PNC-MAC), which is regarded as an extension to the IEEE 802.11 MAC protocol, was developed. PNC-MAC is applicable for PNC with bidirectional flows and without transmission rate and power adaptation. The basic idea of PNC-MAC is to encourage instructive interference which can be used for PNC and, at the same time, avoid destructive interference that may result in packet losses. Nodes randomly access the channel as in the conventional IEEE 802.11 MAC. When there is an opportunity to perform PNC, PNC-MAC coordinates the source nodes to transmit simultaneously. In the cases where PNC is not applicable, PNC-MAC automatically switches back to conventional network coding (CNC) or other conventional relaying schemes, such as plain routing (PR). The performance gains shown in the abovementioned work are mainly from either theoretical analysis or preliminary experiments, and many complicated application scenarios have not been considered. The cross-layer collaboration of 
physical, MAC, and network layers should be more efficient and more adaptive to varying environments in the real world.

The necessity of such collaboration is not difficult to understand; however, some new technical challenges arise in the context of general multi-hop networks. This is particularly true when cooperative communications among nodes are expected to support multiple relaying methods (e.g. PNC, CNC, and PR), multiple traffic patterns (e.g. unidirectional and bidirectional) and multiple data rates with suitable transmission powers. In this article, we address these new challenges by introducing a MAC-centric cross-layer collaboration mechanism for PNC, which encompasses signal superposition, scheduling, and data flow routing. The mechanism for PNC lays a foundation for other physical-layer communication techniques that need to coordinate multiple transmitters and work cooperatively with other protocol layers.

The rest of this article is organized as follows. We first discuss the issues that should be considered during the design of the cross-layer collaboration mechanism for PNC. We then present a general cross-layer protocol architecture and identify the main components and their collaboration in between. Afterwards, the PNC-based packet exchange procedure is described in details, and we evaluate the performance of the cross-layer collaboration mechanism. Finally, we conclude this article and outline directions for future work.

\section{DESIGN CONSIDERATIONS}

The main task of the MAC-centric cross-layer collaboration mechanism is to improve cooperative communication performance between nodes via appropriate relaying operation and adaptive resource management. Since PNC has more stringent compatibility requirements than $\mathrm{CNC}$ and PR, the questions on whether, where, when, and how to utilize PNC so that it is beneficial for the network performance have to be considered in the cross-layer mechanism design. The specific issues are described next.

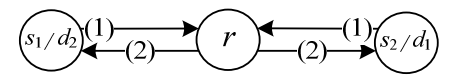

(a) PNC with bidirectional flows.

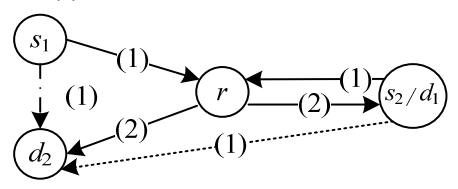

(b) PNC with "Y" flow pattern.

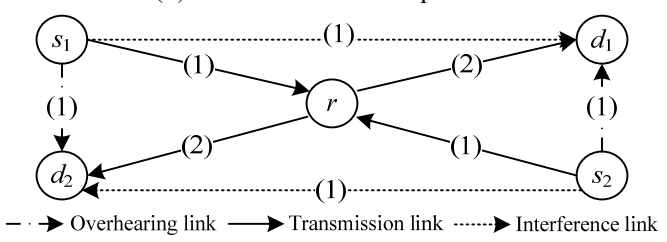

(c) PNC with "X" flow pattern.

Fig. 1. PNC based on three typical topologies. The number in the brackets indicates the transmission timeslot. The variables $s_{1}$ and $s_{2}$ represent source nodes, $d_{1}$ and $d_{2}$ represent destination nodes, and $r$ represents a relay node.

\section{Multiple Traffic Patterns}

The opportunity of performing network coding is partly restricted by the traffic pattern; more specifically, the direction of the actual data flows.

In the case where PNC is performed for bidirectional flows (as shown in Fig. 1(a)), each source is also the destination of the other source. In the first timeslot, the source nodes transmit their packets simultaneously to the relay, which is called as multiple access (MA) phase. In the second timeslot, the superposed signal is mapped into a new symbol sequence as an encoded packet, and the new symbol sequence is forwarded to the destinations over a broadcast channel, which is called as broadcast (BC) phase. Thus, the destination can decode the intended packet from the encoded packet using the copy of its previously transmitted packet.

When using PNC with unidirectional flows, one source node and the destination node of the other source node do not overlap, hence the packet from the source node should be overheard by its neighboring destination node in order to decode the encoded symbols. The "Y" and "X" flow patterns as shown in Fig. 1 (b) and (c) are two typical examples with unidirectional flows, where the two flows are $s_{1} \rightarrow r \rightarrow d_{1}$ and $s_{2} \rightarrow r \rightarrow d_{2}$. In fact, the "X" flow pattern in Fig. 1(c) can also cover the other two patterns by assuming that virtual overhearing links with infinite channel gains exist. Therefore, the following descriptions on the cross-layer collaboration mechanism mainly focus on the "X" traffic pattern.

According to the traffic pattern sensed at the network layer, the cross-layer collaboration mechanism should be able to preliminarily judge whether it is possible to perform $\mathrm{PNC}$ or $\mathrm{CNC}$, and coordinate the transmissions of the involved nodes.

\section{Multiple Data Delivery Methods}

PNC is not always possible to perform, or it may not be the best relaying method. Compared with $\mathrm{CNC}$, PNC may suffer from high bit-error-rate (BER) due to unavoidable self-interference at $d_{1}$ and $d_{2}$ or non-ideal signal superposition at $r$. Moreover, when overhearing links are in low quality, any type of network coding (including PNC and $\mathrm{CNC}$ ) may bring a smaller throughput than PR. Even if all the channel states are good enough, the relay may assume some source nodes have packets to send but they actually do not, due to obsolete information which might be caused by packet loss. Therefore, the cross-layer collaboration mechanism should be able to support other relaying methods (i.e. $\mathrm{CNC}$ and $\mathrm{PR}$ ) besides $\mathrm{PNC}$, and determine the best available relaying method for each relay according to the actual channel conditions measured at the physical layer and traffic patterns obtained at the network layer.

\section{Coordination and Collision}

As shown in Fig. 1(c), the signals sent from $s_{1}$ and $s_{2}$ need to be respectively overheard by $d_{2}$ and $d_{1}$, a superposed signal needs to be received by the relay $r$, and the encoded packet should be correctly decoded by $d_{1}$ and $d_{2}$. Hence, the MAC protocol needs to ensure that there is no interference 
in the neighborhood of $d_{1}, d_{2}$, and $r$, and an appropriate collision avoidance mechanism is necessary. Even if external interference is avoided, the signals sent simultaneously by source nodes $\left(s_{1}\right.$ and $\left.s_{2}\right)$ may interfere with the overheard signals at destination nodes $\left(d_{2}\right.$ and $d_{1}$, respectively). Therefore, coordination between nodes should be guided by the MAC protocol to avoid external collisions; and meanwhile, transmission powers should be adjusted adaptively in the physical layer to minimize the internal interference.

\section{Exceptional Cases}

Because at least three nodes are simultaneously involved in the PNC process, packet loss happens more frequently than in other relaying methods. For the exceptional cases that PNC cannot be performed as expected, some special treatments need to be taken. The treatments, such as abnormality judgment, relaying method switching, and network allocation vector (NAV) resetting, may be triggered in the MAC layer and completed in cooperation with its neighboring layers. Furthermore, these treatments should be conducted timely and correctly.

\section{Transmission Rate and Power Adaptation}

Since channel qualities vary with node location and wireless environment, it would be beneficial if the data rate can be adapted based on channel quality. Meanwhile, the transmission powers of nodes need to be adjusted in order to reduce the interference caused to other nodes. Transmission power adaptation is particularly important in the MA phase of PNC with overhearing, where the two simultaneously transmitting source nodes interfere with each other at the destination nodes. Higher transmission power of one source node, e.g. $s_{1}$, means stronger interference at the other source node's listener $d_{1}$, who needs to overhear the signal from $s_{2}$.

Thus, a distributed MAC protocol that supports rateadaptive cooperative transmissions (including PNC, CNC, and PR) is needed [6]. Optimizing transmission powers and data rates within a single hop is not enough. More efforts are needed to develop optimization schemes for situations involving more than one hop, even the whole network.

\section{PNC-Supported Cross-layer Protocol ARCHITECTURE}

To meet the abovementioned requirements, we introduce a cross-layer protocol architecture, as shown in Fig. 2. By cooperating with the physical and network layers, the MAC layer supports relaying method selection, data information exchange, and exception handling.

In multi-hop wireless networks, an available relaying method needs to be selected when two nodes cannot communicate with each other directly. In our architecture, the most appropriate relaying method among PR and coding-based relaying methods (including $\mathrm{CNC}$ and $\mathrm{PNC}$ ) is selected, subject to additional constraints such as channel conditions, traffic patterns, QoS requirements, etc. A coding opportunity can be sensed based on network connectivity information in the network layer and queuing information in the MAC layer. Whether the sensed coding opportunity is taken as the final relaying method depends on how much throughput it can bring. The relaying method selection module in the MAC layer predicts the throughputs that can be achieved by different relaying method candidates under the current channel conditions, and it selects the relaying method that provides the maximum throughput.

In the relaying method selection process, control information is exchanged between nodes to provide channel quality information. Additionally, the control information exchange assists synchronization and transmission power and rate adaptation in the physical layer.

After deciding the relaying method and the corresponding transmission powers of the involved nodes, the relay node informs the source nodes to transmit data. The MAC protocol coordinates the transmission and assigns the channel resources properly.

When the selected relaying method cannot be performed successfully due to unexpected events (such as channel estimation error, outdated traffic information, etc.), the exception handling module has to react timely and efficiently. One common method is to switch to another relaying method that is capable of completing the transmission task [7].

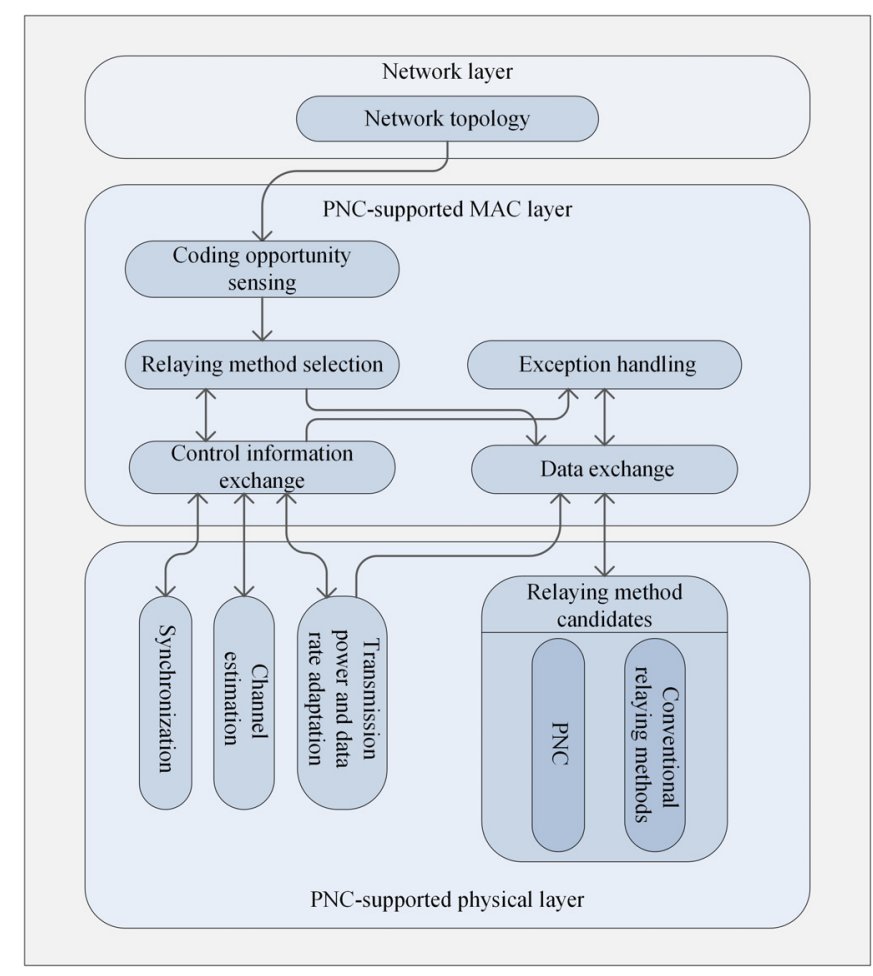

Fig. 2 PNC-supported cross-layer protocol architecture.

\section{Packet Exchange Process in PNC-Supported MAC LAYER}

In this section, we briefly describe how the MAC layer works together with its neighboring layers to support PNC adaptively by modifying the traditional request-tosend/clear-to-send (RTS/CTS) based IEEE 802.11 MAC protocol. Readers can refer to [5] and [8] for more details regarding how to perform frame and signal control in MAC protocols. Fig. 3 presents the standard timing diagram of the 


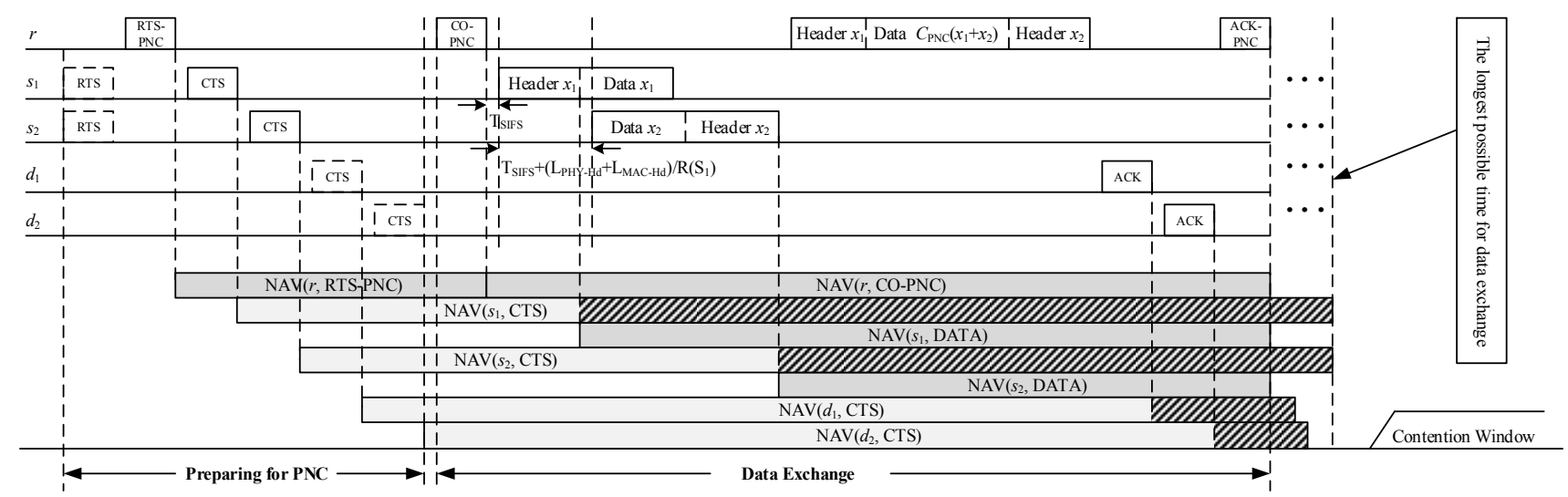

Fig. 3. Standard timing diagram of packet exchange in PNC-supported MAC. The dashed area indicates that the corresponding parts of NAV are set initially but removed after receiving a subsequent packet from the same node.

packet exchange process. Let $T_{\mathrm{SIFS}}$ be the short inter-frame space (SIFS) as in the IEEE 802.11 standard [8]. Let $L_{\mathrm{PHY}-\mathrm{Hd}}$ and $L_{\mathrm{MAC}-\mathrm{Hd}}$ be the length of physical-layer header and MAC-layer header, respectively. The transmission rate of the data packet sent by $s_{1}$ is defined as $R\left(s_{1}\right)$. An underlying routing protocol is assumed, where the network topology within the two-hop range of each node can be sensed. Each node notifies its queue status to its neighboring nodes by adding a few bytes of control information to the data and acknowledgement (ACK) frames.

\section{Sensing PNC Opportunities}

Since multiple relaying methods are supported, each node needs to judge whether PNC is possible under the current network condition. To sense PNC opportunities, each node needs to keep some information regarding the packets that will be forwarded by itself and a list of its neighbors' neighbors. The information of packets to be forwarded is recorded in virtual queues [5]. Take the relay $r$ as an example, each element in its virtual queue has four fields: 1) previous hop, i.e. the source node that currently holds the packet; 2) next hop, i.e. the destination node to which the packet will be forwarded by the relay $r ; 3$ ) length of the packet; 4) holding time, i.e. the time for which the packet has remained in the previous hop. If a first-come, firstserved (FCFS) scheduling policy is used, a PNC opportunity exists when the next hop of one element is either the previous hop or the neighboring node of the previous hop of another element in the virtual queue, and vice versa. The existence of PNC opportunity does not imply that PNC will be used as the final relaying method, because it may not be the best choice among all the available relaying methods. It is also possible that PNC is found to be infeasible in the subsequent procedures, which will be described later.

\section{Preparing for PNC}

When PNC opportunity is sensed, we first assume that PNC is used as the relaying method, and perform some preparations. These preparations include initiating a PNC request, further judging the feasibility and optimality of
PNC, and deciding the optimal transmission rate and power for PNC.

The PNC request can be initiated in two ways, as shown in Fig. 3. Either, the relay $r$ broadcasts a RTS-PNC frame to the neighboring nodes that will be involved in the future PNC process; or, one of the source nodes send a RTS frame to initiate data transmission and the relay $r$ responds by sending a RTS-PNC frame instead of a CTS frame. The nodes that are involved in the PNC operation and receive the RTS-PNC frame will separately send their CTS frames to the relay $r$. For simplicity, the RTS-PNC and CTS frames are sent at the lowest transmission rate, because they are usually much shorter than data frames and optimizing their transmission rates may not bring much performance gain.

The feasibility of PNC is further judged by the relay according to the number of received CTS frames, as well as the status of the involved nodes and channel conditions which are carried by the CTS frames. A CTS frame is called valid when it tells that either the corresponding source node indeed has packets to send or the corresponding destination node can successfully overhear. If the number of valid CTS frames received by the relay $r$ is equal to the number of nodes involved in the possible PNC opportunity, the PNC opportunity is assured.

However, PNC may not be the best relaying method under the current channel conditions. Therefore, whether to perform PNC needs to be further judged according to throughput analysis. Some factors, such as transmission power, BER, channel conditions, modulation level, etc., are all or partly considered during throughput analysis according to application requirements. From this analysis, the optimal data rates and transmission powers for each available relaying method can also be obtained. Finally, the relaying method that is predicted to provide highest throughput is selected.

In the above process, two main types of exceptions exist: 1) the number of valid CTS frames received by $r$ is less than expected; 2) PNC is not selected as the relaying method after throughput analysis. In both cases, other relaying methods (i.e. CNC or PR) will be performed for data exchange. 


\section{Data Exchange}

Data packets are exchanged with the optimal rate and power, and receptions are acknowledged by the receiving nodes. The details of the process are described next.

A coordination (CO-PNC) frame is broadcasted by $r$ to coordinate packet transmissions of $s_{1}$ and $s_{2}$, which contains information regarding whether PNC should be adopted and the transmission rates and powers that should be used by $s_{1}$, $s_{2}$, and $r$.

When CO-PNC is successfully received and PNC is adopted, $s_{1}$ transmits its data frame at data rate $R\left(s_{1}\right)$ after $T_{\mathrm{SIFS}}$, and $s_{2}$ starts its transmission after $2 T_{\mathrm{SIFS}}+\left(L_{\mathrm{PHY}-\mathrm{Hd}}+\right.$ $\left.L_{\mathrm{MAC}-\mathrm{Hd}}\right) / R\left(s_{1}\right)$ and transmits in a bit-reversed order, which means that $s_{2}$ sends the tail of its data frame at first and the header at last. The node holding the shorter packet sends first, and the two packets are transmitted at the same rate. As a result, a time difference between the two data frames ensures that $r$ can successfully decode the headers of both packets sent by the source nodes [5]. If a destination has to overhear packets, it is set to the promiscuous mode after receiving the CO-PNC frame. After successful overhearing, this destination node switches back to the normal mode.

Relay $r$ receives a partly superposed signal sent by $s_{1}$ and $s_{2}$, and broadcasts the resulting partly coded packet to the destinations $d_{1}$ and $d_{2}$. Then, each destination node attempts to decode the coded packet by using the copy of its overheard/own packet. If the intended packet is extracted from the coded packet by the destination, an ACK frame is responded to $r$. After receiving the ACK frames from the destination nodes, an ACK-PNC frame is broadcasted to the sources $s_{1}$ and $s_{2}$ by $r$ to finish this PNC round.

If PNC is not selected as the relaying method, data exchange is performed with CNC or PR. When RTS-PNC has been sent for the current transmission round but PNC is not appropriate to use according to later judgments, the data exchange stage of the timing diagram in Fig. 3 is modified to accommodate CNC or PR [7]. Otherwise, when RTSPNC has not been sent and CNC or PR is selected directly, the reliable broadcasting method as proposed in [9] is used for $\mathrm{CNC}$ and the conventional IEEE 802.11 MAC is used for PR.

\section{NAV Setting and Updating}

The length of the NAV is carried in the duration field of each frame and used to reserve channel. When nodes other than $s_{1}, s_{2}, d_{1}, d_{2}$ and $r$ receive the frames sent during the packet exchange process, they update their NAV timers and remain silent until the time specified by the NAV timers expire. Different NAV length carried by different frames is shown in Fig. 3.

Before the relaying method and transmission rates are decided (i.e. in the stage of preparing for PNC), the NAVs of the CTS frames are temporarily set as the case where the PR relaying method is used and all transmissions are at the lowest rate, to guarantee that the temporary NAVs are not shorter than the time of the whole transmission process.

In the data exchange stage, based on the selected relaying method and transmission rates, the NAV length in the CO-PNC frame is set to precisely cover the remaining time used for data exchange. Generally, the NAV length will be shorter than that in the CTS frame.

In conventional MAC protocols, one node updates its own NAV timer when the NAV length specified by the received frame is larger than the current NAV setting. However, we cannot update the NAV setting in the same way for our PNC-supported MAC protocol discussed in this article because the NAV length may need to be reduced. To resolve this problem, each node maintains a NAV table, which is indexed by node address. Each entry in the NAV table is an individual timer representing the corresponding node. Every time when receiving a new packet, the corresponding entry in NAV table at the receiving node is updated with the latest NAV setting from the sender (no matter whether it increases or decreases the NAV length). The node does not transmit until all NAV timers in the table expire. Recall that the source and destination nodes initially set the NAV length to the largest possible value in their CTS frames. These can be shortened subsequently in data frames and ACK frames, where the NAV lengths are set precisely to the time needed for transmission. This is possible because the relaying method has been determined at this point. When using PNC, the headers of the partly superposed data frame can be separately decoded, hence $s_{1}$ and $s_{2}$ can specify their NAV lengths individually.

\section{Performance Evaluation}

The performance of the cross-layer collaboration mechanism is evaluated on our discrete-event simulator which has detailed physical-layer modeling and supports all the operations in the physical, MAC, and network layers. The simulator is developed jointly with MATLAB and C. We consider the following mechanisms in the simulations: 1) supporting three relaying methods (including PNC, CNC, and PR) with constant transmission powers and data rates (named as PNC-CNC-PR-const); 2) supporting three relaying methods (including $\mathrm{PNC}, \mathrm{CNC}$, and $\mathrm{PR}$ ) with adaptive transmission powers and data rates (named as PNC-CNC-PR-adapt); 3) supporting two relaying methods (including $\mathrm{CNC}$ and $\mathrm{PR}$ ) with constant transmission powers and data rates (named as CNC-PR-const); 4) supporting two relaying methods (including $\mathrm{CNC}$ and $\mathrm{PR}$ ) with adaptive transmission powers and data rates (named as CNC-PRadapt); 5) only supporting PR with constant transmission powers and data rates (named as PR-const); 6) only supporting PR with adaptive transmission powers and data rates (named as PR-adapt). The rate and power adaptation method developed in [10] is used. A random topology is considered, where 14 random flows exist among 20 nodes that are randomly distributed in an $800 \times 800 \mathrm{~m}^{2}$ area.

The throughput performances of these mechanisms are shown in Fig. 4. We can observe that the PNC-CNC-PRadapt mechanism outperforms other mechanisms. It implies that the PNC-oriented cross-layer collaboration mechanism can significantly improve throughput via adaptive transmission and resource allocation based on traffic pattern and network conditions. We can also observe that the PNCCNC-PR-const mechanism performs better than other mechanisms that also have constant powers and rates. It demonstrates that PNC can improve throughput, especially 
with the efficient collaboration between physical layer and upper layers. We can also find that the CNC-PR-adapt mechanism has the lowest throughput among all the mechanisms with rate and power adaption, which is caused by unnecessary overhead for coding opportunity sensing [9].

The end-to-end delay performances of these mechanisms are shown in Fig. 5. We can observe that the end-to-end delays of the PNC-supported mechanisms are similar as or slightly higher than those of the PNC-excluded mechanisms. The reason is that unsuccessful PNC operations due to contentions at bottleneck nodes cause relaying method switching at the cost of delay. However, when we compare PNC-CNC-PR-adapt with PR-adapt, the throughput gain is much higher than the delay increases. We can therefore still conclude that PNC with adaptive rate and power control is beneficial, particularly for throughputdemanding applications, such as file transfer.

In summary, the performance gain in terms of delay using PNC-CNC-PR-adapt is much better than those of most other methods, while the throughput gain is definitely the best when PNC-CNC-PR-adapt is adopted

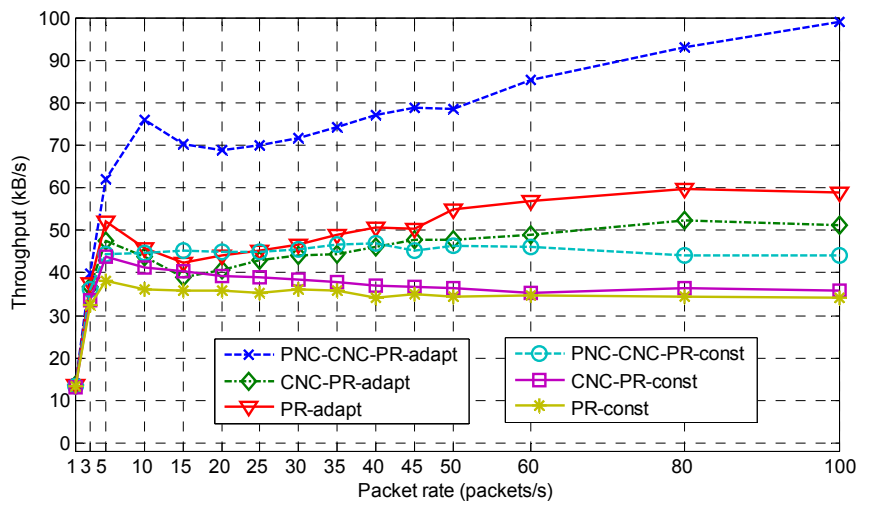

Fig. 4 Throughput comparison between different transmission mechanisms.

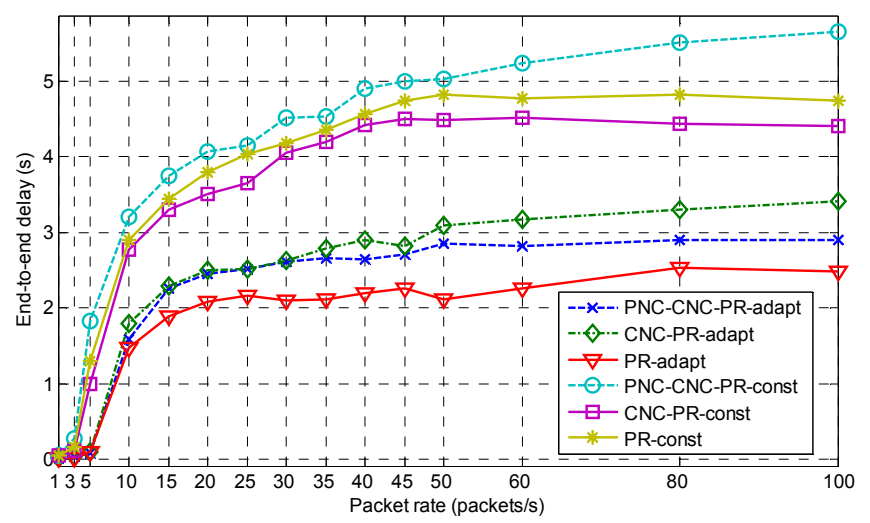

Fig. 5 End-to-end delay comparison between different transmission mechanisms.

\section{CONCLUSION AND FUTURE WORK}

The emerging physical-layer techniques for cooperative communications are promising for improving spectrum resource utilization. In order to further explore the efficient implementation of these techniques, in this article, we have taken PNC as an example and presented a MAC-centric cross-layer collaboration mechanism for PNC. By leveraging cross-layer coupling, the information collected from different layers is synthesized to help wireless nodes to coordinate efficiently and adaptively. The idea of crosslayer collaboration for PNC presented in this article can be applied to other similar physical-layer techniques and has the potential to advance the development of cooperative communications.

In future research, information and requirements in upper layers (e.g. application layer) should be considered to deepen the cooperation between nodes. Especially, crosslayer information exchange between the bottom three layers and the application layer should be reinforced so that users' preferences collected in the application layer can timely help the bottom three layers to optimize the cooperation via reasonable resource assignment and appropriate utilization of physical-layer techniques. Additionally, the tradeoff between performance and complexity in the cross-layer collaboration mechanism design for emerging physicallayer techniques should be considered. Efforts should be made to reach the design goal effectively by a simple way with low signaling overhead.

\section{ACKNOWLEDGMENT}

Lei Guo was the corresponding author for this paper. This work was supported in part by the National Natural Science Foundation of China (61172051, 61302072, 91438110, 61471109), the Fundamental Research Funds for the Central Universities (N110804003, N120804002, N120404001), the Program for New Century Excellent Talents in University (NCET-12-0102), and the Specialized Research Fund for the Doctoral Program of Higher Education (20120042120049).

\section{REFERENCES}

[1] S. Zhang, S. C. Liew, and P. P. Lam, "Hot topic: physical-layer network coding," in Proc. ACM MOBICOM 2006, Sept. 2006, pp. 358-365.

[2] A. Akhtar, M. Nakhai, A. Aghvami, "On the Use of Cooperative Physical Layer Network Coding for Energy Efficient Routing", IEEE Transactions on Communications, vol. 61, no. 4, April 2013, pp. 1498-1509.

[3] S. Katti, S. Gollakota, and D. Katabi, "Embracing wireless interference: analog network coding," in Proc. SIGCOMM 2007, Aug. 2007, pp. 397-408.

[4] M. Khabbazian, F. Kuhn, N. Lynch, M. Medard, and A. ParandehGheibi, "MAC design for analog network coding," MIT Computer Science and Artificial Intelligence Laboratory Technical Report, Aug. 2010.

[5] S. Wang, Q. Song, X. Wang, and A. Jamalipour, "Distributed MAC protocol supporting physical-layer network coding," IEEE Transactions on Mobile Computing, vol. 12, no. 5, May 2013, pp. $1023-1036$

[6] A. Argyriou, "Coordinating Interfering Transmissions in Cooperative Wireless LANs", IEEE Transactions on Wireless Communications, vol. 10, no. 11, Nov. 2011, pp. 3804-3812.

[7] F. Wang, Q. Song, S. Wang, L. Guo, and A. Jamalipour, "MAC protocol supporting physical-layer network coding with overhearing," in Proc. IEEE GLOBECOM 2013, Dec. 2013, pp. 5128-5133.

[8] M. Gast, 802.11 Wireless Networks: The Definitive Guide, Second Edition. Sebastopol: O’Reilly Media, Inc., 2005. 
[9] A. Argyriou, "Wireless network coding with improved opportunistic listening," IEEE Trans. on Wireless Communications, vol. 8, no. 4, Apr. 2009, pp. 2014-2023.

[10] F. Wang, Q. Song, S. Wang, and L. Guo, "Rate and Power Adaptation for Physical-Layer Network Coding with $M$-QAM Modulation", in Proc. IEEE ICC 2014, Jun. 2014, pp. 5519-5524.

\section{BIOGRAPHIES}

Qingyang Song received the $\mathrm{PhD}$ degree in telecommunications engineering from the University of Sydney, Australia. She is an associate professor at Northeastern University, China. She has authored more than 50 papers in major journals and international conferences. These papers have been cited more than 900 times in scientific literature. Her current research interests are in radio resource management, cognitive radio networks, cooperative communications, ad-hoc networks, heterogeneous cellular networks, and protocol design. She is a senior member of the IEEE.

Lei Guo is a Professor at the Northeastern University, Shenyang, China. He received the Ph.D. degree from University of Electronic Science and Technology of China, China, in 2006. He His research interests include communication networks, optical communications and wireless communications. He has published over 200 technical papers in the above areas on international journals and conferences, such as IEEE TCOM, IEEE TWC, IEEE/OSA JLT, IEEE/OSA JOCN, IEEE GLOBECOM, IEEE ICC, etc. Dr. Guo is a member of the IEEE and the OSA, and he is also a senior member of CIC. He is now serving as an editor for five international journals, such as Photonic Network Communications, The Open Optics Journal, etc.

Fanzhao Wang received the BEng degree from Northeastern University, China, in 2011. He is currently working toward the $\mathrm{PhD}$ degree in the School of Information Science and Engineering, Northeastern University, China. His interests include full-duplex communications, cooperative communications, protocol design, and optimization algorithms.

Shiqiang Wang received the BEng and MEng degrees from Northeastern University, China, respectively in 2009 and 2011 . He is currently working toward the $\mathrm{PhD}$ degree in the Department of Electrical and Electronic Engineering, Imperial College London, United Kingdom. He spent the Summer 2014 and Summer 2013 at IBM Thomas J. Watson Research Center, Yorktown Heights, NY, United States; and the Autumn 2012 at NEC Laboratories Europe, Heidelberg, Germany. His research interests include dynamic control mechanisms, optimization algorithms, protocol design and prototyping, with applications to mobile cloud computing, hybrid and heterogeneous networks, vehicular networks, ad-hoc networks, and cooperative communications. He has approximately 20 scholarly publications, and has served as a technical program committee member or reviewer for several international journals and conferences.

Abbas Jamalipour (S'86-M'91-SM'00-F'07) is the Professor of Ubiquitous Mobile Networking at the University of Sydney, Australia, and holds a PhD in Electrical Engineering from Nagoya University, Japan. He is a Fellow of the Institute of Electrical, Information, and Communication Engineers (IEICE) and the Institution of Engineers Australia, an ACM Professional Member, and an IEEE Distinguished Lecturer. He is the author of six technical books, nine book chapters, over 300 technical papers, and three patents, all in the area of wireless communications. $\mathrm{He}$ was the Editor-in-Chief IEEE Wireless Communications and has been an editor for several journals. He is the Vice President-Conferences (2012-13) and a member of Board of Governors of the IEEE Communications Society. Previously he has held positions of the Chair of the Communication Switching and Routing and the Satellite and Space Communications Technical Committees and Vice Director of the Asia Pacific Board, in ComSoc. He was a General Chair or Technical Program Chair for several IEEE ICC, GLOBECOM, WCNC and PIMRC conferences. Dr. Jamalipour is also an elected member of Board of Governors (2014-16), IEEE Vehicular Technology Society. He is the recipient of a number of prestigious awards such as the 2010 IEEE ComSoc Harold Sobol Award, the 2006 IEEE ComSoc Distinguished Contribution to Satellite Communications Award, the 2006 IEEE ComSoc Best Tutorial Paper Award. 